\title{
Some certain properties of the generalized hypercubical functions
}

\author{
Duško Letić ${ }^{1}$ Nenad Cakić ${ }^{2}$, Branko Davidović ${ }^{3 *}$, Ivana Berković ${ }^{1}$ and Eleonora Desnica ${ }^{1}$
}

\footnotetext{
* Correspondence: iwtbg@beotel. net

${ }^{3}$ Technical High School, Kragujevac, Serbia

Full list of author information is available at the end of the article
}

\begin{abstract}
In this article, the results of theoretical research of the generalized hypercube function by generalizing two known functions referring to the cube hypervolume and hypersurface and the recurrent relation between them have been presented. By introducing two degrees of freedom $k$ and $n$ (and the third half-edge $r$ ), we are able to develop the derivative functions for all three arguments and discuss the possibilities of their use. The symbolic evaluation, numerical experiment, and graphic presentation of the functions are realized using Mathcad Professional and Mathematica.
\end{abstract}

MSC 2010: 33E30; 33E50; 33E99; 52B11.

Keywords: special functions, hypercube function, derivate

\section{Introduction}

The hypercube function ( $\mathrm{HC}$ ) is a hypothetical function connected with multidimensional space. It belongs to the group of special functions, so its testing is being performed on the basis of known functions of the type: $\Gamma$-gamma, $\psi$-psi, $l n$-logarithm, exp-exponential function, and so on. By introducing two degrees of freedom $k$ and $n$, we generalize it from discrete to continual [1,2]. In addition, we can advance from the field of the natural integers of the dimensions-degrees of freedom of cube geometry, to the field of real and non-integer values, where all the conditions concur for a more condense mathematical analysis of the function $H C(k, n, r)$. In this article, the analysis is focused on the infinitesimal calculus application of the $\mathrm{HC}$ which is given in the generalized form. For research papers on the development of multidimensional function theory, see Bowen [3], Conway [4], Coxeter [5], Dewdney [6], Hinton [7], Hocking and Young [8], Gardner [9], Manning [10], Maunder [11], Neville [12], Rucker [13], Skiena [14], Sloane [15], Sommerville [16], Wilker [17], and others and for its testing, see Letić et al. [18]. Today the results of the HC research are represented both in geometry and topology and in other branches of mathematics and physics, such as Boole's algebra, operational researches, theory of algorithms and graphs, combinatorial analysis, nuclear and astrophysics, molecular dynamics, and so on.

\section{The derivative HCs}

\subsection{The hypercube functional matrix}

The former results [2], as it is known, give the functions of the hypercube surface $(n=2)$, i.e., volume $(n=3)$, therefore, we have, respectively

(c) 2011 Letićć et al; licensee Springer. This is an Open Access article distributed under the terms of the Creative Commons Attribution License (http://creativecommons.org/licenses/by/2.0), which permits unrestricted use, distribution, and reproduction in any medium, provided the original work is properly cited. 


$$
H C(k, 2, r)=2 k r^{k-1}=\frac{\partial}{\partial r} H C(k, 3, r) \text { or } H C(k, 3, r)=\int_{0}^{r} H C(k, 2, r) d r=(2 r)^{k} .
$$

On the basis of the above recurrent relations, we formulated the general form of the $\mathrm{HC}[1]$.

Definition 2.1. The generalized HC is defined by equality

$$
H C(k, n, r)=\frac{2^{k} r^{k+n-3} \Gamma(k+1)}{\Gamma(k+n-2)} \quad(k, n \in \Re, \quad r \in N) .
$$

where $r$ is the half-edge of the hypercube. These functions need to be the functions of three variables with two degrees of freedom $k$ and $n$ and the hypercube radius $r$. With real cubic entities there exist, for example, square edge, length, size, or surface, then the cube surface and volume, where there exists only the variable-the half-edge $r$ (Figure 1).

Having in mind the characteristic that the derivatives with respect to the half-edge $r$ generate new functions (the $H C$ matrix columns), we perform "movements" to lower or higher degrees of freedom. We start from the $n$th degree of freedom, on the basis of the following recurrent relations:

$$
\frac{\partial}{\partial r} H C(k, n, r)=H C(k, n-1, r) \text { and } H C(k, n+1, r)=\int_{0}^{r} H C(k, n, r) d r .
$$

The previous characteristics are essential and hypothetical. They also hold for elements outside of this submatrix of six elements (see Figures 1 and 2). For example, the derivations $(2.3$, left) show that we have obtained the zeroth $(n=0)$ degree of freedom,

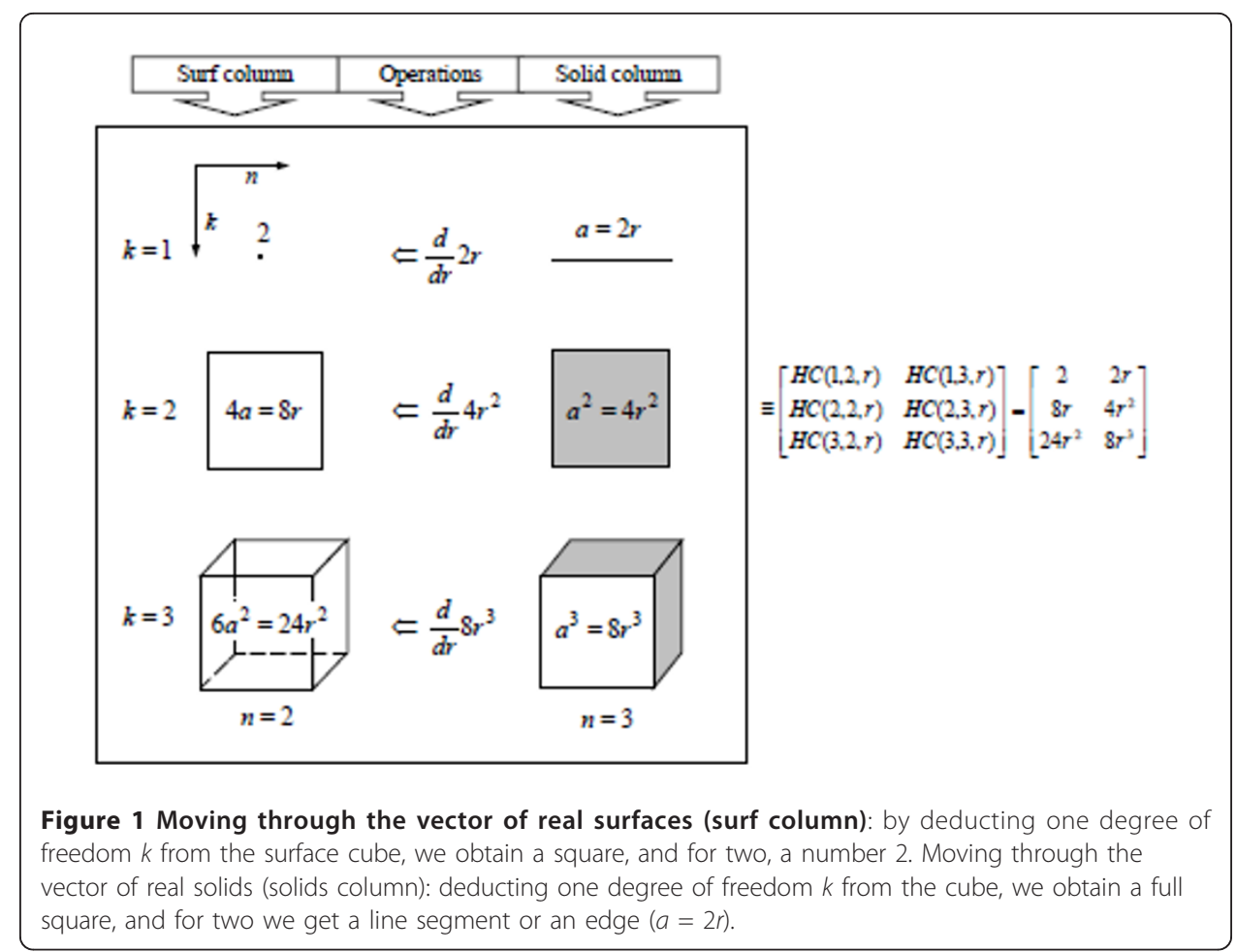




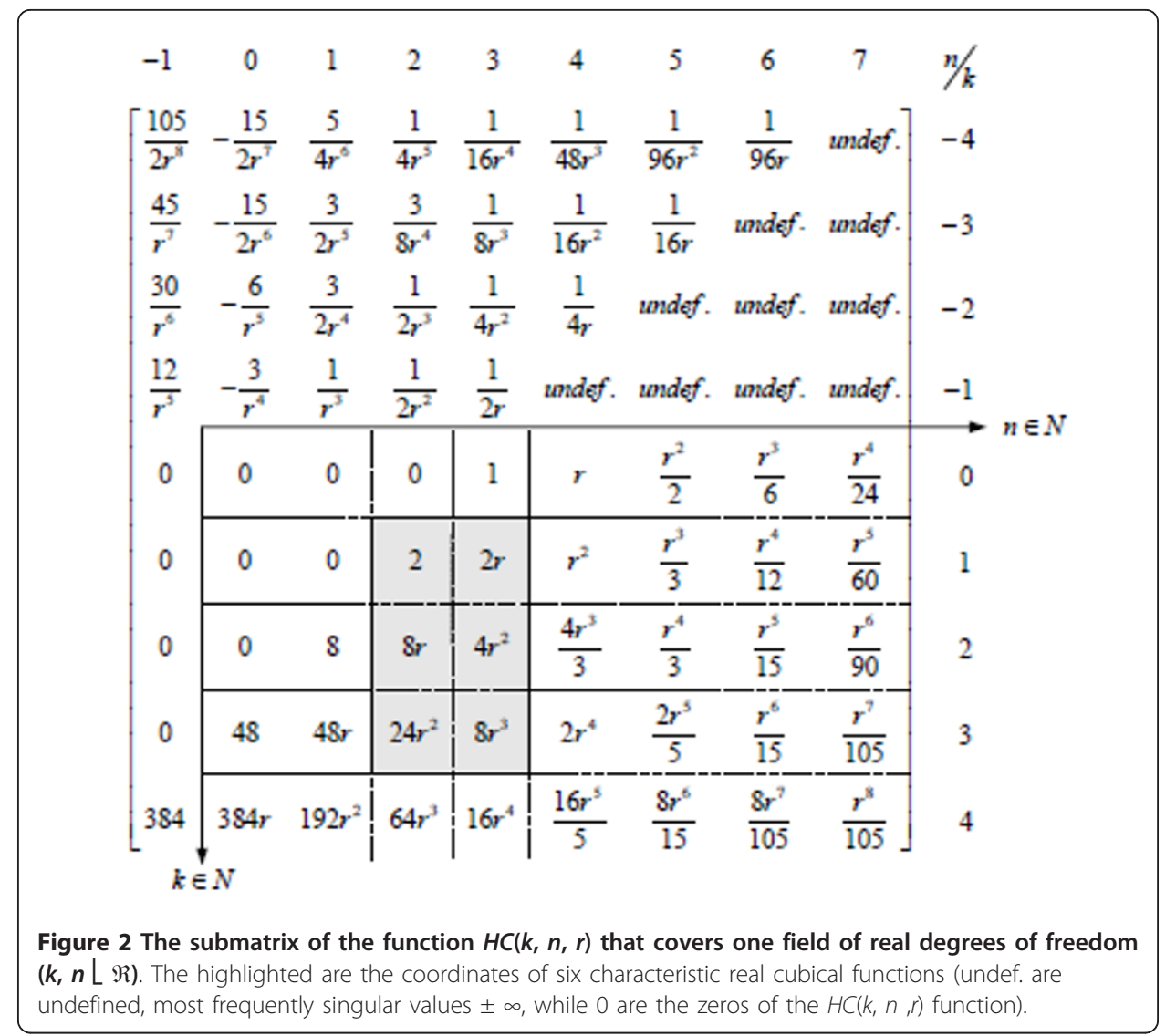

if we perform the $n$th degree derivation. Using the defined derivative degree, the $H C$ function is being calculated as well for the complex field of the hypercube matrix, namely, we obtain an expression that is equal to (2.3, right)

$$
\frac{\partial^{n}}{\partial r^{n}} H C(k, n, r)=H C(k, 0, r) \text { or } \frac{\partial^{2 n}}{\partial r^{2 n}} H C(k, n, r)=H C(k,-n, r) .
$$

Due to the known characteristics of the gamma function and on the basis of relations generalizing (2.2) and (2.3), we obtain the following differential equation:

$$
\frac{\partial^{n}}{\partial r^{n}} \sum_{k=0}^{\infty} H C(k, n, r)-\sum_{k=0}^{\infty} H C(k, 0, r)=0 \text { or } \frac{\partial^{2 n}}{\partial r^{2 n}} \sum_{k=0}^{\infty} H C(k, n, r)-\sum_{k=0}^{\infty} H C(k,-n, r)=0 .
$$

So, on the basis of the recurrent relation, we establish a connection with the positive degree of freedom $n$ and its symmetrical negative degree $(-n)$, through the differential equation which describes the relation among the columns of the hypercube matrix for $k, n \in \mathfrak{R}(2.4)$. In view of the general HC (2.1), we develop an adequate matrix $M[H C]$ $k x n(k, n \in \mathfrak{R})$, where concrete values for the selected submatrix $9 \times 9$ follow as well.

$$
M[H C]_{k x n}=
$$


For example when $n=3$ and $k=\overline{1,4}$ we obtain the following relation

$$
\frac{\partial^{4}}{\partial r^{4}} H C(k, 3, r)=H C(k,-1, r) \text { and } \frac{\partial^{4}}{\partial r^{4}}\left[\begin{array}{c}
2 r \\
4 r^{2} \\
8 r \\
8 r^{2} \\
16 r^{4}
\end{array}\right]=\left[\begin{array}{c}
0 \\
0 \\
0 \\
0 \\
384
\end{array}\right] .
$$

The matrix $M[H C]_{k x n}$ is based on the characteristic that each of its vectors of the ( $n-1$ )-column (also marked as $\langle n-1>$ ) is equal to the derivative with respect to the half-edge $r$ of the following vector $(<n>)$ and in the order according to Figure 2. This recursive characteristic ordinates among the initial assumptions (2.2).

Theorem 2.2. From the columns of the matrix $[M]_{k x w}$ the following equality holds:

$$
[M]^{<n-1>}=\frac{\partial}{\partial r}[M]^{<n>} .
$$

For example, for two adjacent columns of the matrix $[M]^{<n-1>}$ and $[M]^{<n>}$, the recurrent vectors follow

$$
[M]^{<n-1>}=\frac{\partial}{\partial r}\left[\begin{array}{c}
\frac{r^{n-3}}{\Gamma(n-2)} \\
\frac{2 r^{n-2}}{\Gamma(n-1)} \\
\frac{8 r^{n-1}}{\Gamma(n)} \\
\frac{48 r^{n}}{\Gamma(n+1)} \\
\vdots \\
\frac{2^{k} r^{k+n-3} \Gamma(k+1)}{\Gamma(k+n-2)}
\end{array}\right]=\left[\begin{array}{c}
\frac{r^{i-n}}{\Gamma(n-1)} \\
\frac{2 r^{n-3}}{\Gamma(n)} \\
\frac{8 r^{n-2}}{\Gamma(n+1)} \\
\frac{48 r^{n-1}}{\Gamma(n+2)} \\
\vdots \\
\frac{2^{k} r^{k+n-4} \Gamma(k+1)}{\Gamma(k+n-1)}
\end{array}\right] \text { for } n \in \Re .
$$

Interesting results can be obtained on the basis of horizontal ( $n$ ) or vertical ( $k$ ) degrees of freedom (arranged in 2.4) and the generalized HC function, e.g., for the level surface, as follows:

$$
\left.\left.\left.k 2^{k} r^{k-1}\right|_{k=3} \vee \frac{8 r^{n-1}}{\Gamma(n)}\right|_{n=2} \vee \frac{2^{k} r^{k+n-3} \Gamma(k+1)}{\Gamma(k+n-2)}\right|_{k=3 \wedge n=2} \Rightarrow 24 r^{2} \equiv 6 a^{2},
$$

or for the solid level

$$
\left.\left.\left.(2 r)^{k}\right|_{k=3} \vee \frac{48 r^{n}}{\Gamma(n+1)}\right|_{n=3} \vee \frac{2^{k} r^{k+n-3} \Gamma(k+1)}{\Gamma(k+n-2)}\right|_{k=3 \wedge n=3} \Rightarrow 8 r^{3} \equiv a^{3} .
$$

This characteristic is very significant, because we can obtain the same result in view of the two special formulae, or using only one, the general.

\subsection{The analysis if the recurrent potential function of the type $z^{v}$}

The HC, besides the gamma function, also has the potential component $r^{k+n-3}$. The generalized equation of the $h$ th derivation of the fractional power function $z^{v}$ amounts to $[19]$

$$
\frac{\partial^{h} z^{v}}{\partial z^{h}}=\frac{\Gamma(v+1)}{\Gamma(v-h+1)} z^{v-h} \quad(-v \notin N) .
$$


Namely, here we know that the exponent with the basis of the half-edge $r$ of the $H C$ function is $v=k+n-3$. Now, the $m$ th derivation of the $\mathrm{HC}$ on the radius is defined by the following equation

$$
\frac{\partial^{m}}{\partial r^{m}} H C(k, n, r)=\frac{2^{k} \Gamma(k+1)}{\Gamma(k+n-2)} \cdot \frac{\partial^{m} r^{k+n-3}}{\partial r^{m}},
$$

so, the $m$ th derivation is reduced to

$$
\frac{\partial^{m}}{\partial r^{m}} H C(k, n, r)=\frac{1}{r^{m}} \frac{\Gamma(k+n-2)}{\Gamma(k+n-m+1)} H C(k, n, r)
$$

After applying some transformations on the above expression, we obtain the function form.

Theorem 2.3. The $m$ th derivative of the hypercubical function with respect to the radius $r$ is

$$
\frac{\partial^{m}}{\partial r^{m}} H C(k, n, r)=H C(k, n-m, r)=\frac{2^{k} r^{k+n-m-3} \Gamma(k+1)}{\Gamma(k+n-m-2)} .
$$

originating from the known characteristic $\Gamma(z+1)=z \Gamma(z)$. Equation 2.5 is recurrent by nature, and with it we find every degree of the expression (for $+m)$ and integral (for $-m)$, depending on the position of (n) for which we do these operations. In that sense we define and, where appropriate, use a unique operator with which we merge the operations of differentiating and integrating (using the unique symbol $D^{ \pm m}$ ) on the radius of the HC function. These operations are generalized as well on non-integer (fractional) degrees of derivative/integral.

Definition 2.4. The unique operator that merges the operations of differentiating and integrating with respect to the radius of the hypercubical function is given by

$$
\frac{\partial^{ \pm m}}{\partial r^{ \pm m}} H C(k, n, r) \equiv D^{ \pm m}\{H C(k, n . r)\}
$$

The defined integrals of the $H C$ function are (with the reference degree of freedom $v$ $=k+n-3)$ equal to

$$
\begin{aligned}
& \int_{0}^{r} H C(k, n, r) d r=\frac{r}{v+1} H C(k, n, r)=H C(k, n+1, r), \\
& \int_{0}^{r} \int_{0}^{r} H C(k, n, r) d r d r=\frac{r^{2}}{(v+1)(v+2)} H C(k, n, r)=H C(k, n+2, r) \\
& \vdots \\
& \underbrace{\int_{0}^{r} \int_{0}^{r} \cdots \int_{0}^{r} H C(k, n, r) d r d r \cdots d r}=\frac{r^{m}}{(v+1)(v+2) \ldots \quad(v+m)} H C(k, n, r)=H C(k, n+m, r) .
\end{aligned}
$$


The $H C$ function multiplier can be presented in a product form in this shape

$$
\frac{r^{m}}{(v+1)(v+2)(v+3) \cdots(v+m)}=r^{m} \prod_{i=0}^{m-1} \frac{1}{(v+i+1)}
$$

or

$$
r^{m} \prod_{i=0}^{m-1} \frac{1}{(v+i+1)}=\frac{r^{m} \Gamma(v+1)}{\Gamma(v+m+1)}
$$

so the integral of the $m$ th degree is defined as

$$
\underbrace{\int_{0}^{r} \int_{0}^{r} \cdots \int_{0}^{r} H C(k, n, r) d r d r \cdots d r}_{m}=\frac{r^{m} \Gamma(v+1)}{\Gamma(v+m+1)} H C(k, n, r) .
$$

After some transformations, we get a more generalized form

$$
\underbrace{\int_{0}^{r} \int_{0}^{r} \cdots \int_{0}^{r} H C(k, n, r) d r d r \cdots d r}_{m}=\frac{2^{k} r^{k+n+m-3} \Gamma(k+1)}{\Gamma(k+n+m-2)} .
$$

The generalized recurrent relation could symbolically be expressed by the term

$$
\frac{\partial^{ \pm m}}{\partial r^{ \pm m}} H C(k, n, r)=H C(k, n \mp m, r)=\frac{2^{k} r^{k+n \mp m-3} \Gamma(k+1)}{\Gamma(k+n \mp m-2)}
$$

where we assume that

$$
\frac{\partial^{-m}}{\partial r^{-m}} H C(k, n, r)=H C(k, n+m, r)=\underbrace{\int_{0}^{r} \int_{0}^{r} \cdots \int_{0}^{r} H C(k, n, r) d r d r \cdots d r}_{m} .
$$

The general equation covers derivational and integral characteristics of recursion and has the following form

$$
\frac{\partial^{ \pm m}}{\partial r^{ \pm m}} H C(k, n, r)-H C(k, n \mp m, r)=0 .
$$

Having in mind the known characteristics of the gamma function, the value of differential and integral degree $m$ need not be integer, as e.g., with classical differentiating (integrating).

\subsection{Fractional differentials/integrals of the $\mathrm{HC}$ function}

The degree of derivation (or integration) $m$ may be integer or non-integer, consequently, out of the field of real numbers. So, for example, for the integer derivatives the following values are representative and each of them gives the same result.

Example 2.5. In the first case, there exists a second derivative of the $H C$ function connected to the degree of freedom $n=5$ and derivative degree $m=2$, as follows 


$$
\frac{\partial^{2}}{\partial r^{2}} H C(k, 5,2, r)=(2 r)^{2}
$$

or in the second case by double integrating of the $H C$ function, when $n=1$ and $m=-2$, it follows

$$
\frac{\partial^{-2}}{\partial r^{-2}} H C(k, 1,-2, r)=(2 r)^{2}
$$

Both operations give the same result. Applying non-integer (fractional) derivative degrees, e.g., $m= \pm 1 / 2$, starting with the fixed degrees of freedom $n=7 / 2$ and $n=5 / 2$, we obtain the same results as the previous ones, with the procedure of integer differentiating/integrating (2.6) and (2.7). Thus, the results follow

$$
\frac{\partial \frac{1}{2}}{\partial r \frac{1}{2}} H C\left(k, \frac{7}{2}, \frac{1}{2}, r\right)=(2 r)^{2}
$$

in other words with non-integer integralling $(m=-1 / 2)$

$$
\frac{\partial^{-\frac{1}{2}}}{\partial r^{-\frac{1}{2}}} H C\left(k, \frac{5}{2},-\frac{1}{2}, r\right)=(2 r)^{2} \text {. }
$$

Evidently that the results (2.6), (2.7), (2.8), and (2.9) are identical.

\subsection{The HC gradient}

Gradient may be applied on the hypercubical function, taking into consideration its differentiability and multidimensionality. As this function has three variables, $k, n$, and $r$, the solution of gradient functions $\nabla_{k, n, r}$ is given as follows

$$
\nabla_{k, n, r}\{H C(k, n, r)\}=\left[\begin{array}{c}
\frac{\partial}{\partial k} H C(k, n, r) \\
\frac{\partial}{\partial n} H C(k, n, r) \\
\frac{\partial}{\partial r} H C(k, n, r)
\end{array}\right]=H C(k, n, r)\left[\begin{array}{c}
\ln 2 r+\psi_{0}(k+1)-\psi_{0}(k+n-2) \\
\ln r-\psi_{0}(k+n-2) \\
\frac{1}{r}(k+n-3)
\end{array}\right] .
$$

This function is particularly noteworthy with fixing the extreme of the contour $H C$ functions.

\subsection{Contour graphics of HCs}

The graphics of the function and their derivatives may be simply obtained by computer analysis. Each checking in sense of the analytical expanding of the HCs (Figure 3) and in the left half-plane $(k \leq 0)$, it relies on the characteristics of the gamma $(\Gamma)$ and digamma $\left(\psi_{0}\right)$ functions, which make the HC.

The derivation functions $h c 1=d H C / d k=\nabla_{k}\{H C\}$ and $c h 1=d H C / d n=\nabla_{n}\{H C\}$ also belong to the family of HCs. Using them, we separate two groups of functions, according to the degrees of freedom $k$ and $n$. The first derivate function on the degree of freedom $k$ is as follows 


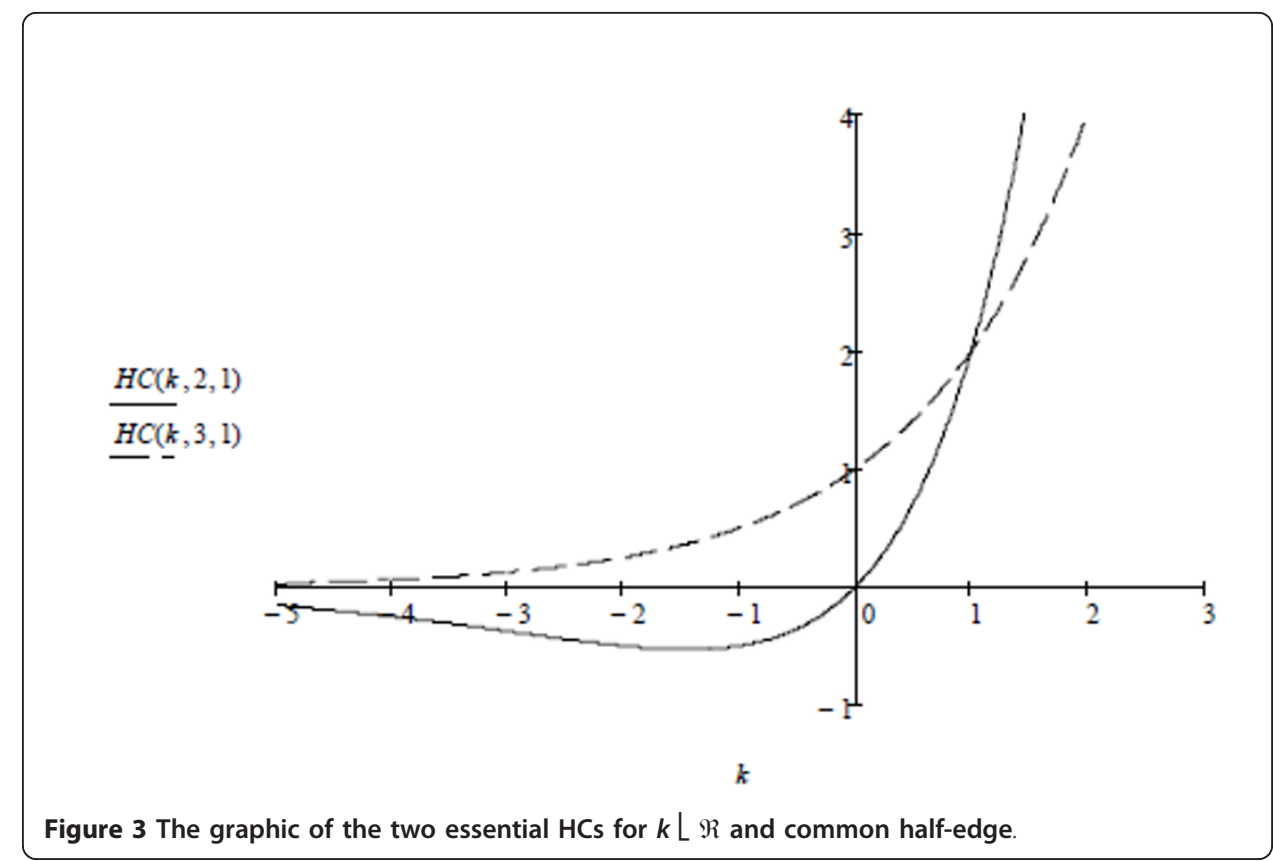

$$
h c 1(k, n, r)=\frac{\partial}{\partial k} H C(k, n, r)=H C(k, n, r)\left[\ln 2 r+\psi_{0}(k+1)-\psi_{0}(k+n-2)\right],
$$

and has a special meaning when we determine the maximum of HCs, e.g., for the common half-edge and the domain $k \in N$ (Figure 4). Also, on the basis of the known

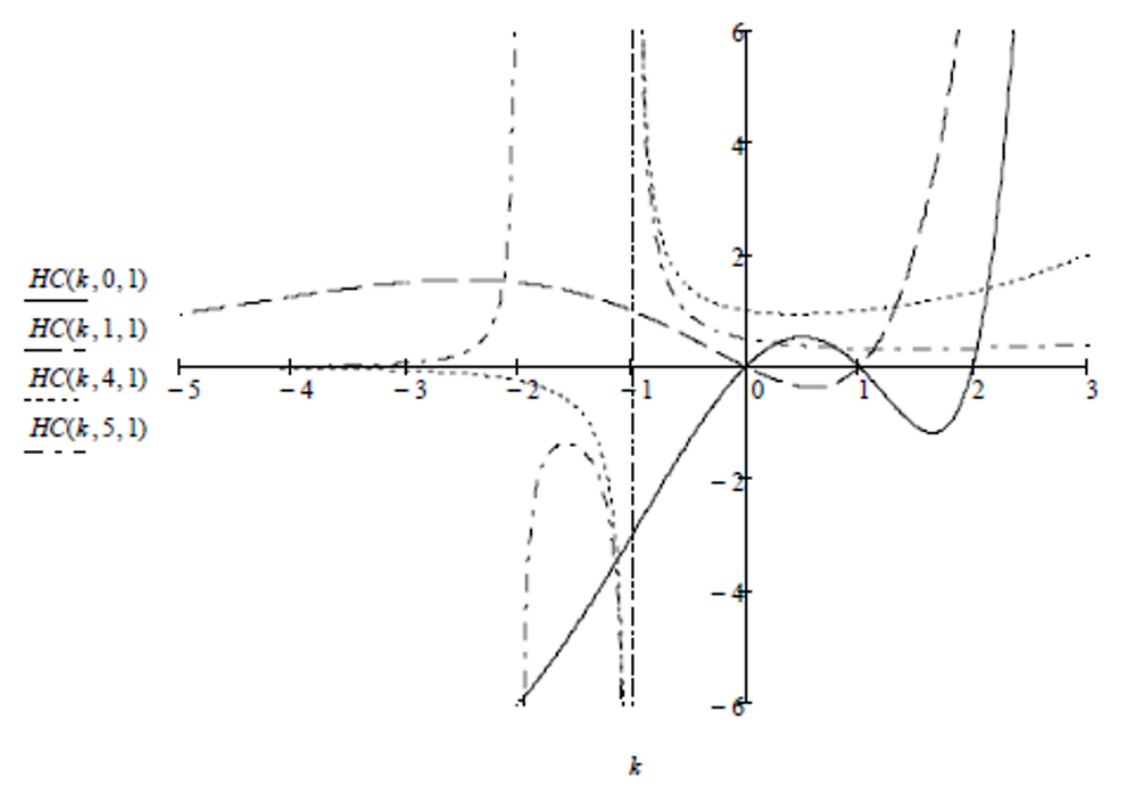

Figure 4 The HCs for the certain values of the degrees of freedom $n=0,1,4,5$ and the values $k L$ $\mathfrak{R}$. 


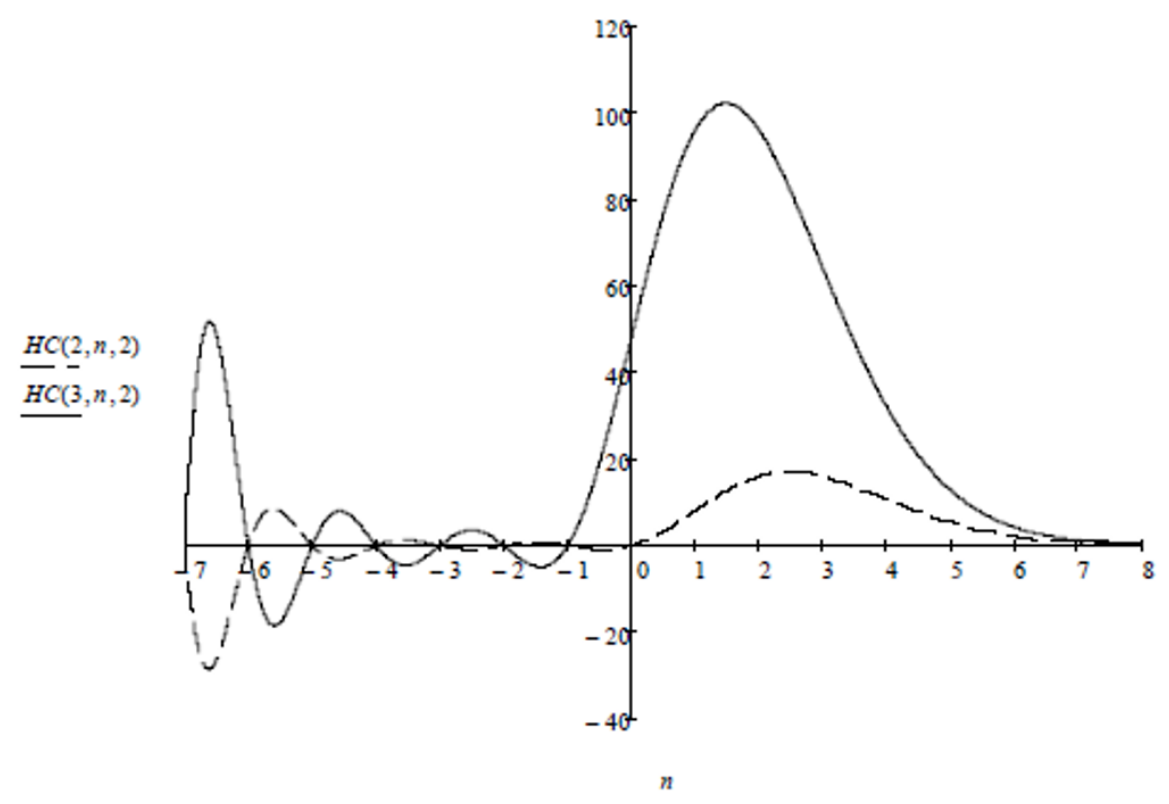

Figure 5 The surface $\mathrm{HC}$ and its derivative function with characteristically values.

criteria, for each function in the family of derivations functions, we define the maximum value of the $H C$ function by equating its derivative with zero. Other than the maximum, we give the following, where the value of the degree of freedom $k_{0}$ is named as "optimal". Consequently

$$
\frac{\partial}{\partial k} H C(k, n, r)=0 \text { and } \frac{\partial^{2}}{\partial k^{2}} H C(k, n, r)>0 \text { and } \frac{\partial^{2}}{\partial k^{2}} H C(k, n, r)>0 \Rightarrow \max H C(k, n, r) \wedge k_{0} .
$$

\subsection{The extreme values of the HC function from the viewpoint of the freedom degree $k$}

The function $h c 1(k, n, r)$ has a special meaning (Figure 5) in defining the extreme values of the HCs, e.g., for the common radius and the domain $k \in \Re$. Also, in view of the known criteria, for each function in the family of derivative functions, we obtain minimum values of the $H C$ functions by equating its derivative with zero. Other than the minimum, we give the following, where the value of the degree of freedom $k_{0}$ is named as "optimal".

$$
h c 1(k, n, r)=0 \text { and } \frac{\partial}{\partial k} h c 1(k, n, r)>0 \Rightarrow \min H C(k, n, r) \wedge k_{0} .
$$

For the surface $\mathrm{HC}$, with the half edge $r=1$, the derivative function, after some transformations, is

$$
h c 1(k, 2,1)=H C(k, 2,1)\left[\ln 2+\psi_{0}(k+1)-\psi_{0}(k-1)\right]=0 .
$$

Taking into consideration $\psi_{0}(k+1)-\psi_{0}(k-1)=\frac{1}{k}$, this expression may be rationalized as 


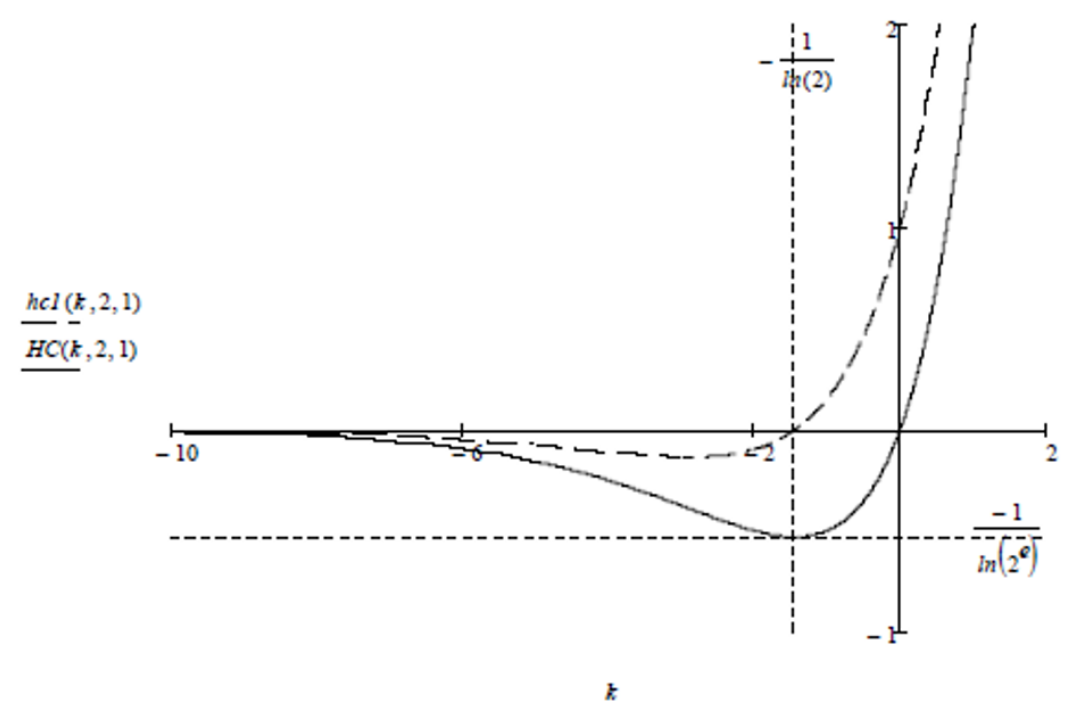

Figure 6 Surface and solid HC for certain degrees of freedom $n L \Re$ and common half-edges (or $a$ $=2$ ).

Example 2.6. By equating with zero, the expression in brackets is

$$
\ln 2+\frac{1}{k}=0 \quad \Rightarrow \quad k_{0}=-\frac{1}{\ln 2} \approx-1,44269
$$

We obtain a symbolical solution for the "optimal" dimension (2.10) (Figure 6).

The symbolical and numerical value states are

$$
H C\left(k_{0}, n, 1\right)=-\frac{1}{\ln 2^{e}} \approx-0,53074 .
$$

On the basis of (2.10)-(2.11), we define the minimum cube surface for the "optimal" dimension $k_{0}$. Also, we obtain $k_{0} \approx-1,44269$, which gives the lateral surface of min $H C$ $\left(k_{0}, n, 1\right) \approx-0,53074$ as in (Table 1$)$. For other HCs, we also define minimum values, but clearly on numeric bases. Meanwhile, for degrees of freedom $n \geq 3$, the minimum

Table 1 Minimum cube surface for optimal dimension and various degrees of freedom

\begin{tabular}{llll}
\hline Dimension $\boldsymbol{n}$ & Optimal dimension $\boldsymbol{k}_{\mathbf{0}}$ & $\min \boldsymbol{H C}$ & Error \\
\hline-1 & 2.680571494 & -6.2786205562 & $4.293 \times 10^{-12}$ \\
0 & 1.648207317 & -1.1780735642 & $1.940 \times 10^{-11}$ \\
1 & 0.584187070 & -0.3641730147 & $-1.836 \times 10^{-12}$ \\
2 & -1.442695041 & -0.5307378454 & $5.191 \times 10^{-13}$ \\
3 & $-\infty$ & 0 & 0 \\
$\ldots$ & $\ldots$ & $\ldots$ & $\ldots$ \\
$i$ & $\ldots$ & 0 & 0 \\
$\ldots$ & $\ldots$ & $\ldots$ & $\ldots$ \\
$n$ & $\ldots$ & 0 & 0 \\
\hline
\end{tabular}


Table 2 Extreme values of contour functions where $\boldsymbol{k}$ is known

\begin{tabular}{llll}
\hline Dimension $\boldsymbol{k}$ & Optimal dimension $\boldsymbol{n}_{\mathbf{0}}$ & $\max \boldsymbol{H C}$ & Error \\
\hline 0 & 3.461632145 & 1.129017388 & $6.619 \times 10^{-15}$ \\
1 & 2.461632145 & 2.258347771 & $3.818 \times 10^{-14}$ \\
2 & 1.461632145 & 9.033391083 & $-3.179 \times 10^{-12}$ \\
3 & 0.461632145 & 54.20034650 & $-2.964 \times 10^{-11}$ \\
4 & 0 & 384 & 0 \\
$\ldots$ & $\ldots$ & $\ldots$ & $\ldots$ \\
$j$ & 0 & $2^{k} \frac{\Gamma(j+1)}{\Gamma(j-2)}$ & 0 \\
$\ldots$ & $\ldots$ & $\ldots$ & $\ldots$ \\
$k$ & 0 & $2^{n} \frac{\Gamma(n+1)}{\Gamma(n-2)}$ & 0 \\
\hline
\end{tabular}

comes down to zero. The greatest values of the considered functions are infinite, when $k_{0}=\infty$.

\subsection{The first derivatives of the $c h 1$ function of $H C$ in relation to the degree of freedom $n$} Since the function $H C(k, n, r)$ is the function of two variables (not taking into consideration the radius $r$ ), we can determine its partial derivatives on the degree of freedom $n$, so the derivatives are set, respectively.

The general solution is

$$
\operatorname{ch} 1(k, n, r)=\frac{\partial}{\partial n} H C(j, n, r)=H C(k, n, r)\left[\ln r-\psi_{0}(k+n-2)\right] .
$$

These functions can be important in further research of the HC. The extreme values of these contour functions $H C(k, n, r)$ (where $k$ is known) can be presented in Table 2, and they are visually shown in Figures 7 and 8 ,

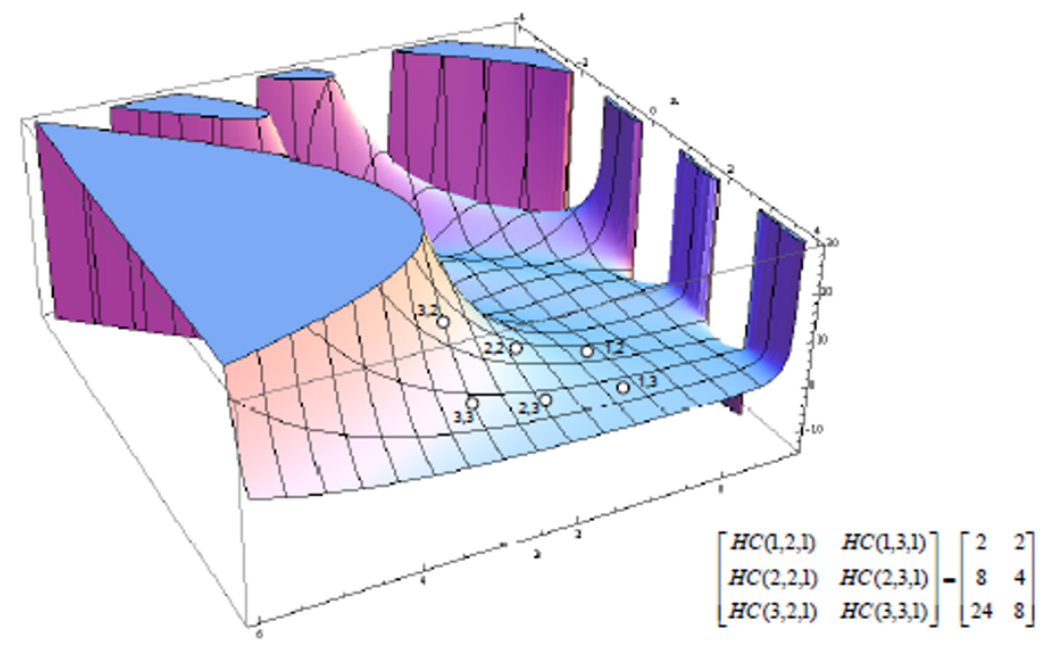

Figure $73 \mathrm{D}$ HC for the unit radius $H C(-1 \leq k \leq 6,-4 \leq n \leq 4,1)=\frac{2^{k} \Gamma(k+1)}{\Gamma(k+n-2)}$ and coordinates of real cubic entities $(k, n\lfloor\Re)$ 


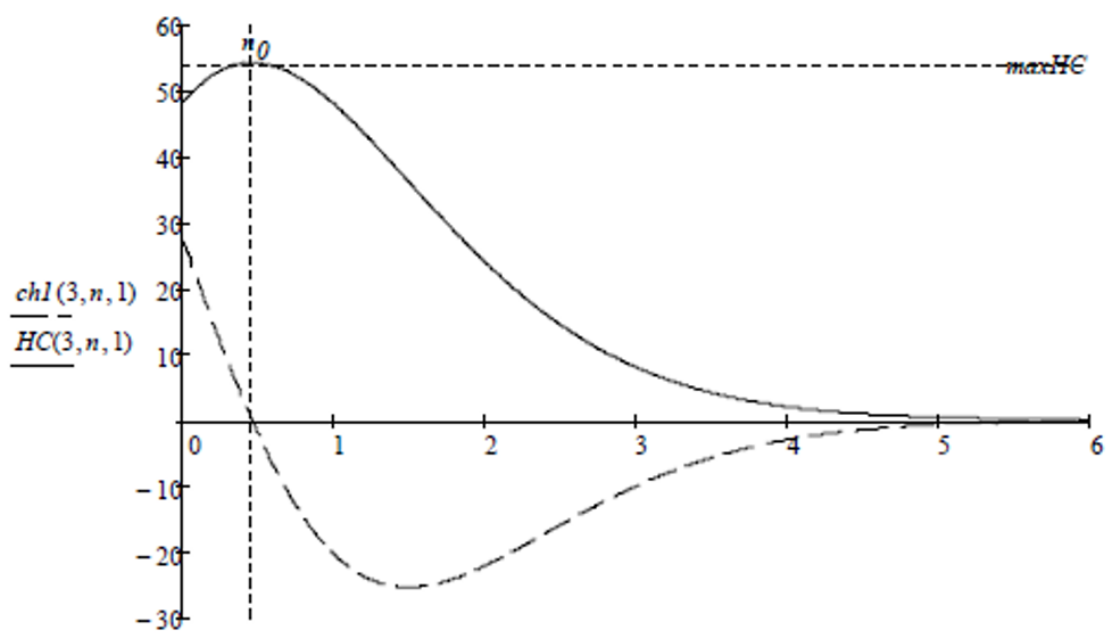

Figure 8 The $\mathrm{HC}$ and its derivative with the $H C$ maximum location and optimum for the value of the degree of freedom $n_{0} \approx 0,46163$ for $n L N$, for the degree of freedom $k=3$ and the common half-edge.

\subsection{The higher-order derivatives of the $\mathrm{HC}$ function on the $k$ argument}

The derivatives of the $\mathrm{HC}$ for any argument retain the characteristics similar to the original $H C$ function. This is especially noticeable in graphical representation. In addition, it would be interesting to examine the regularities in the structure of this function of the higher degree derivation. In that sense, later in this article, we present the derivative functions on the $k$ argument (the more complex case) i.e. $n$ (the simpler case) analytically and graphically. The second derivative of the $\mathrm{HC}$ on the argument $k$ is

$$
\begin{aligned}
& h c 2(k, n, r)=\frac{\partial^{2}}{\partial k^{2}} H C(k, n, r)= \\
& H C(k, n, r)\left\{\left[\psi_{0}(k+1)-\psi_{0}(v+1)+\ln 2 r\right]^{2}+\psi_{1}(k+1)-\psi_{1}(v+1)\right\},
\end{aligned}
$$

where $\psi_{0}(z)=\frac{d}{d z} \ln \Gamma(z)$ is the digamma, $\psi_{1}(z)=\frac{d^{2}}{d z^{2}} \ln \Gamma(z)$ the trigamma function, and $\zeta(z)$ Riemann's zeta function. The concrete value of this derivative for the fixed parameters $k=3$ and $n=3$ is obtained later. The mixed derivation of the $\mathrm{HC}$ is

$$
\begin{aligned}
& \frac{\partial^{2}}{\partial k \partial n} H C(k, n, r)= \\
& H C(k, n, r)\left[\left(\psi_{0}(v+1)-\ln r\right)\left(\psi_{0}(v+1)-\psi_{0}(k+1)-\ln 2 r\right)-\psi_{1}(v+1)\right] .
\end{aligned}
$$

Example 2.7. After applying the same values of $k$ and $n$ it follows that

$$
\left.\frac{\partial^{2} H C(k, n, 1)}{\partial k \partial n}\right|_{\substack{k=3 \\ n=3}}=4\left(2 \gamma-\frac{11}{3}\right) \ln 2-\frac{2}{3}\left(2 \pi^{2}-\frac{46}{3}\right) .
$$

The fourth derivative of the $\mathrm{HC}$ on the argument $n$, and the common radius is of the form 
Here, we include a degree of freedom as $v=n+k-3$.

Example 2.8. For the $\mathrm{HC} H C(k, n, r)$, some values of the derivative function of the argument $n$, are

$$
\begin{aligned}
& \operatorname{ch} 4(0,0,1)=2\left\{4[3 \gamma+2 \zeta(3)]-2 \gamma^{2}(9-2 \gamma)-\pi^{2}(2 \gamma-3)\right\}, \\
& \operatorname{ch} 4(1,2,1)=2 \gamma\left[8 \zeta(3)+\gamma\left(\gamma^{2}-\pi^{2}\right)\right]+\frac{\pi^{2}}{5} \zeta(2), \\
& \operatorname{ch} 4(0,2,1)=8 \zeta(3)+2 \gamma\left(2 \gamma^{2}-\pi^{2}\right), \\
& \operatorname{ch} 4(2,0,2)=8\left[4 \zeta(3)+(\gamma+\ln 2)\left(6 \gamma \ln 2-\pi^{2}\right)+2\left(\gamma^{3}+\ln ^{3} 2\right)\right],
\end{aligned}
$$

where $\zeta(z)$ is the Riemann's zeta function, and $\zeta(3) \approx 1,202057$ the Apery's constant [20].

\section{Conclusion}

When we obtain the $H C$ functions of the $m$ th derivative, we notice a certain regularity in the disposal of coefficients in addition to the polygamma functions $(-1,10,-10,-15$, $5,10,-1$; see (3.1)). Partial derivatives with respect to the argument $k$ are relatively complicated and they have been obtained only for the first and second derivatives. The previous partial derivations of the $H C$ function have been derived to the fifth degree of freedom $n$, so in that case, for $r=1$, we obtain the following coefficients along with psi (polygamma) function (3.1). As it is known, this derivative can be obtained as well on the basis of integral equation, where we consider $m=5$.

$$
\frac{d^{m}}{d n^{m}} \Gamma(n)=\int_{0}^{\infty} t^{n-1} e^{-t}(\ln t)^{m} d t \Rightarrow \frac{d^{5}}{d n^{5}} \Gamma(n)=\int_{0}^{\infty} t^{n-1} e^{-t}(\ln t)^{5} d t .
$$

Such integral presentation of the derivative of reciprocal gamma function, and also $\mathrm{HC}$ is known, but the equivalent solution (3.1) is not explained enough with the alternative members of the psi functions.

$$
\begin{aligned}
& h c 5(k, n, r)=\frac{\partial^{5}}{\partial n^{5}} H C(k, n, r) \\
& =-H C(k, n, r)\left\{\left(\psi_{0}(k+n-2)-\ln r\right)^{5}-10\left(\psi_{0}(k+n-2)-\ln r\right)^{3} \psi_{1}(k+n-2)\right. \\
& +10\left(\psi_{0}(k+n-2)-\ln r\right)^{2} \psi_{2}(k+n-2)+15\left(\psi_{0}(k+n-2)-\ln r\right)^{2} \psi_{1}^{2}(k+n-2) \\
& \left.-5 \psi_{3}(k+n-2)\left(\psi_{0}(k+n-2)-\ln r\right)-10 \psi_{1}(k+n-2) \psi_{2}(v+1)+\psi_{4}(k+n-2)\right\} \\
& =-H C(k, n, r)\left\{\left(\psi_{0}(k+n-2)-\ln r\right)^{5}-10\left(\psi_{0}(k+n-2)-\ln r\right)^{3} \psi_{1}(k+n-2)+\right. \\
& 10\left(\psi_{0}(k+n-2)-\ln r\right)^{2} \psi_{2}(k+n-2)+15\left(\psi_{0}(k+n-2)-\ln r\right)^{2} \psi_{1}^{2}(k+n-2)- \\
& \left.5 \psi_{3}(k+n-2)\left(\psi_{0}(k+n-2)-\ln r\right)-10 \psi_{1}(k+n-2) \psi_{2}(v+1)+\psi_{4}(k+n-2)\right\} \text {. }
\end{aligned}
$$

In general, the $\mathrm{HC}$ of the $m$ th derivative of $n$ can be defined on the basis of the product of polygamma (psi) polynomial of the $m$ th degree and HC. Its general form is the following

$$
\operatorname{chm}(k, n, 1)=\frac{\partial^{m}}{\partial n^{m}} H C(k, n, r)=H C(k, n, r) \cdot f^{m}\left\{\psi_{(m-1)}(k+n-2), r\right\} .
$$


The analysis of the multidimensional object-cube and the formulas of this geometrysuggests its complexity and connection with special functions and the other fields of geometry. Certain issues (for which we do not give solutions in this article) relate to the rational and general forms of the derivative on the two degrees of freedom, while the general derivative/integral on the argument $r$ is obtained in (2.1) and it amounts to

$$
\frac{\partial^{ \pm m}}{\partial r^{ \pm m}} H C(k, n, r)=\frac{2^{k} r^{k+n \mp m-3} \Gamma(k+1)}{\Gamma(k+n \mp m-2)}
$$

\title{
Author details
}

${ }^{1}$ Technical Faculty "M. Pupin", University of Novi Sad, 23000 Zrenjanin, Serbia 2Faculty of Electrical Engineering, University of Belgrade, 11000 Belgrade, Serbia ${ }^{3}$ Technical High School, Kragujevac, Serbia

\begin{abstract}
Authors' contributions
DL worked on defining hyperspherical, hypercylindrical, and hypercube functions and matrix and its graphical representation, and defining the diagonal fluxes of these functions. NC a professor who teaches special functions defined by the terms and conditions under which these functions existed especially in complex areas. BD allowed the individual chapters of these works are unified as they work independently on these functions includes about 500 pages. IB provided input regarding the software verification function, its derivative, the calculation of fluxes and the like. ED was primarily engaged in 2D and 3D graphics and visualization functions and their zeros and examining many singularities. All of the previous team of mathematicians and computer specialists who are now in the stage of the global project in which the target set as a generalization hyperspherical, hypercylindrical and hypercube function (Archimedean bodies) on a new basis through special functions. The project currently considered about 1650 pages and is divided into 7 volumes of scientific monographs of which one is published, and one is in the final review. All team members agree to the idea that these research results published in reputable scientific journals. The most important work that is under construction "refers to the generalization of hyper-squaring the circle". All authors read and approved the final manuscript.
\end{abstract}

\section{Competing interests}

The authors declare that they have no competing interests.

Received: 4 May 2011 Accepted: 6 December 2011 Published: 6 December 2011

\section{References}

1. Letić, D, Cakić, D, Davidović, B: Conjecture about hypercubical function, (monograph to prepare). Technical Faculty M. Pupin, Zrenjanin. (2010)

2. Weisstein, EW: Hypercube. From MathWorld-A Wolfram Web Resource. http://mathworld.wolfram.com/Hypercube.html

3. Bowen, JP: Hypercubes. Practical Comput. 5(4), 97-99 (1982)

4. Conway, JH: Sphere Packing, Lattices and Groups. pp. 9. Springer-Verlag, New York, 2 (1993)

5. Coxeter, HSM: Regular Polytopes. Dover, New York, 3 (1973)

6. Dewdney, AK: Computer a program for rotating for hypercubes indices four-dimensional dementia. Sci Am. 254, 14-23 (1986)

7. Hinton, $\mathrm{CH}$ : The Fourth Dimenzion. Health Research, Pomeroy. (1993)

8. Hocking, JG, Young, GS: Topology. Dover, New York (1988)

9. Gardner, M: Hypercubes, in Mathematical Carnival: A New Round-Up of Tantalizers and Puzzles from Scientific. pp. 4154. Vintage Books, New YorkCh. 4, (1977)

10. Manning, H: Geometry of Fourth Dimension. Dover, New York (1956)

11. Maunder, CRF: Algebraic Topology. Dover, New York (1997)

12. Neville, EH: The Fourth Dimension. Cambridge University Press, Cambridge (1921)

13. Rucker, R, Von, B: The Fourth Dimension: A Guided Tour of the Higher Universes, Houghtson Miffin, Boston. (1984)

14. Skiena, S: Hypercubes, in Implementing Discrete Mathematics: Combinatorics and Graph Theory with Mathematica. pp. 148-150. Addison-Wesley, Reading (1990) ?\$?4.2.5

15. Sloane, NJA: Sequences A000079/M1129, A001787/M3444, A001788/M4161, A001789/M4522, and A091159 in The OnLine Encyclopedia of Integer Sequences'.http://oeis.org/

16. Sommerville, DMY: An Introduction to the Geometry of $n$ Dimensions. pp. 136. Dover, New York (1958)

17. Wilker, JB: An extremum problem for hypercubes. J Geom. 55, 174-181 (1996). doi:10.1007/BF01223043

18. Letić, D, Cakić, N, Ramanujan, S: The Prince of Numbers. Computer Library, Belgrade (2010). (ISBN 976-86-7310-452-2)

19. Power Function: Differentiation (subsection 20/03/01).http://functions.wolfram.com/ElementaryFunctions/Power/20/03/ $01 /$

20. Letić, D, Cakić, D, Davidović, B: Mathematical Constants-Exposition in Mathcad. Beograd (2010). (ISBN 978-86-87299-047)

doi:10.1186/1687-1847-2011-60

Cite this article as: Letic et al.: Some certain properties of the generalized hypercubical functions. Advances in

Difference Equations 2011 2011:60. 